Check for updates

Cite this: Mater. Horiz., 2021, 8,2513

Received 9th June 2021, Accepted 12th July 2021

DOI: $10.1039 / \mathrm{d} 1 \mathrm{mh} 00908 \mathrm{~g}$

rsc.li/materials-horizons

With the emergence of stretchable/wearable devices, functions, such as sensing, energy storage/harvesting, and electrical conduction, should ideally be carried out by a single material, while retaining its ability to withstand large elastic deformations, to create compact, functionally-integrated and autonomous systems. A new class of trimodal, stretchable yarn-based transducer formed by coating commercially available Lycra ${ }^{\circledR}$ yarns with PEDOT:PSS is presented. The material developed can sense strain (first mode), and temperature (second mode) and can power itself thermoelectrically (third mode), eliminating the need for an external power-supply. The yarns were extensively characterized and obtained an ultrahigh (gauge factor $\sim 3.6 \times 10^{5}$, at $10-20 \%$ strain) and tunable (up to about 2 orders of magnitude) strain sensitivity together with a very high strain-at-break point (up to $~ 1000 \%$ ). These PEDOT:PSS-Lycra yarns also exhibited stable thermoelectric behavior (Seebeck coefficient of $15 \mu \mathrm{V} \mathrm{K}{ }^{-1}$ ), which was exploited both for temperature sensing and self-powering $(\sim 0.5 \mu \mathrm{W}$, for a 10-couple module at $\Delta T \sim 95 \mathrm{~K})$. The produced material has potential to be interfaced with microcontroller-based systems to create internet-enabled, internet-of-things type devices in a variety of form factors.

Stretchable and wearable sensors are attracting an ever increasing interest, in response to demand from fields like soft (micro-) robotics, ${ }^{1,2}$ smart textiles, ${ }^{3,4}$ electronic skins ${ }^{5,6}$ and health monitoring devices. ${ }^{7-10}$ Although high flexibility ${ }^{11-13}$ and sensing capability

${ }^{a}$ School of Engineering and Materials Science, Queen Mary University of London, Mile End Road, London, E1 4NS, UK. E-mail: e.bilotti@qmul.ac.uk

${ }^{b}$ Department of Materials, Loughborough University, Loughborough, LE11 3TU, UK

${ }^{c}$ Nanoforce Technology Ltd., Joseph Priestley Building, Queen Mary University of London, Mile End Road, London, E1 4NS, UK

${ }^{d}$ Department of Bioengineering, Imperial College London, London SW7 2AZ, UK

${ }^{e}$ Institute for Materials Discovery, University College London, Malet Place, London WC1E 7JE, UK

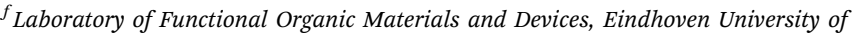
Technology, P.O. Box 513, 5600 MB Eindhoven, The Netherlands

${ }^{g}$ School of Physics and Astronomy, Queen Mary University of London, Mile End Road, London, E1 4NS, UK

$\dagger$ Electronic supplementary information (ESI) available. See DOI: 10.1039/ d1mh00908g

\section{New concepts}

In this work, we provided a novel concept to fabricate a new class of trimodal, stretchable transducers. The fabricated transducer can sense strain (first mode), and temperature (second mode) and can power itself thermoelectrically (third mode). This yarn-based transducer differentiates itself from existing literature as it exhibits the highest combined values of sensitivity and stretchability reported for self-powered strain sensors. Thanks to the insight acquired on the sensing mechanism of functional coating, we also demonstrated that the strain sensitivity could be adjusted by a simple processing parameter, namely the pre-strain value, in a wide range and for different target values. Moreover, the general applicability of the fabrication concept could be extended to a broad range of materials and hence properties, by coating different classes of thermoelectrical materials on various elastic/soft materials. As a proof of concept, we also demonstrated a wireless movements' detector and a proof-of-concept glove, which can harvest body energy and sense the environment and object temperature in a self-powered mode.

to different stimuli ${ }^{14-18}$ have been demonstrated, a number of critical constraints still limit the practical use of flexible sensors. One of the key challenges is how to retain functions (e.g. ability to sense, energy storage/harvesting, electrical/thermal conductivity) at large deformations. For example, strain sensors with either high gauge factor (GF) (up to 1000-16 000) and low strain at break $(2-7 \%)^{19,20}$ or, vice versa, with a wide range of working strain (up to 280-300\%) but low GF (0.06-13.1), have been reported. ${ }^{21-23}$ Recently, this challenge has been partially addressed by incorporating auxetic mechanical metamaterials (GF $\sim 800$ and strain-at-break of $160 \%),{ }^{24}$ or by patterning silver nanowires on polydimethylsiloxane films (GF $\sim 150000$ and strain-at-break of $60 \%){ }^{25}$

Beyond the sensitivity/deformation dichotomy, the need for (typically rigid) energy storage devices is another critical constraint limiting the practical use of flexible and highly deformable sensors. Developing autonomous systems that are able to generate enough power in situ using, for instance, the photovoltaic, ${ }^{26-28}$ piezoelectric, ${ }^{29}$ triboelectric, ${ }^{30,31}$ and thermoelectric (TE) effect, ${ }^{12,32-35}$ could be a solution. Thermoelectricity 
allows harvesting of energy from ubiquitous temperature differences, such as body temperature to ambient temperature, in a reliable and consistent manner.

Fibre-based sensors have attracted particular attention in wearable applications. They possess many features, such as high surface area, good flexibility and recoverable deformation. However, fibre-based strain sensors usually have very low electrical conductivity because the addition of sufficiently high conductive filler content can significantly affect their spinnability and mechanical properties. The conflict between the mechanical properties and electrical conductivities also hinders the development of a stretchable temperature sensor. Moreover, the low electrical conductivities limited the thermoelectrical efficient so that no self-powered strain/temperature sensor based on yarn has been reported.

We present, for the first time, an ultrasensitive self-powered smart yarn, based on the thermoelectric properties of PEDOT:PSS (poly(3,4-ethylene-dioxythiophene)-poly(styrene sulfonate)) coated on a commercial Lycra ${ }^{\circledR}$ yarn (detailed in $\left.\mathrm{ESI} \dagger^{36}\right)$. By imposing and tuning cracks in the PEDOT:PSS coating layer, we achieve a unique combination of large deformations and ultrahigh sensitivity. Moreover, the sensor could be autonomously powered by the thermoelectric response of the PEDOT:PSS coating, when subjected to a temperature gradient (e.g. $20 \mathrm{~K})$, and detect, for instance, cyclic strains and temperature (difference).

The ultra-sensitive smart yarn was fabricated by a swellcoating method (Fig. 1A), which involves the application of a PEDOT:PSS layer via dip-coating over the surface of a preswelled multifilament Lycra ${ }^{\mathbb{R}}$ (commercial polyurethane) yarn. Pre-swelling in dimethyl sulfoxide (DMSO) and the additional presence of DMSO in the aqueous PEDOT:PSS coating suspension, generates a more robust PEDOT:PSS coating layer, which results in a significantly increased electrical conductivity (Fig. S1, ESI $\dagger$ ) and improved water-stability (Fig. S2, ESI $\dagger$ ). A unique surface morphology, with a wrinkled PEDOT:PSS coating layer, was formed during the drying process, due to the difference in volumetric shrinkage between PEDOT:PSS and Lycra ${ }^{\mathbb{R}}$ yarns as well as the large mismatch in their stiffness values (the Young's modulus of PEDOT:PSS and Lycra is 1500 MPa and 7.5 MPa, respectively). Although the shrinkage takes place in both longitudinal and transverse direction of the yarn (Fig. S3, ESI $\dagger$ ), transvers wrinkles (i.e. perpendicular to the yarn's axial direction) are much more evident than along the other direction (Fig. 1B). This is believed due to the larger axial deformation taking place during the drying and shrinkage process, as a result of the yarn's geometry (i.e. large aspect ratio) and its anisotropy (i.e. PU molecules are aligned along the yarn direction), which is induced by the spinning and stretching production process. A relatively good interfacial adhesion between the PEDOT:PSS layer and the polyurethane substrate was observed (Fig. 1C), attributed to the formation of hydrogen bonds between the oxygen-containing groups in the PEDOT:PSS and the $\mathrm{N}-\mathrm{H}$ groups of polyurethane yarns. The interfacial shear stress is estimated at around 1.4 MPa by using the critical length observed from SEM images and by fitting the
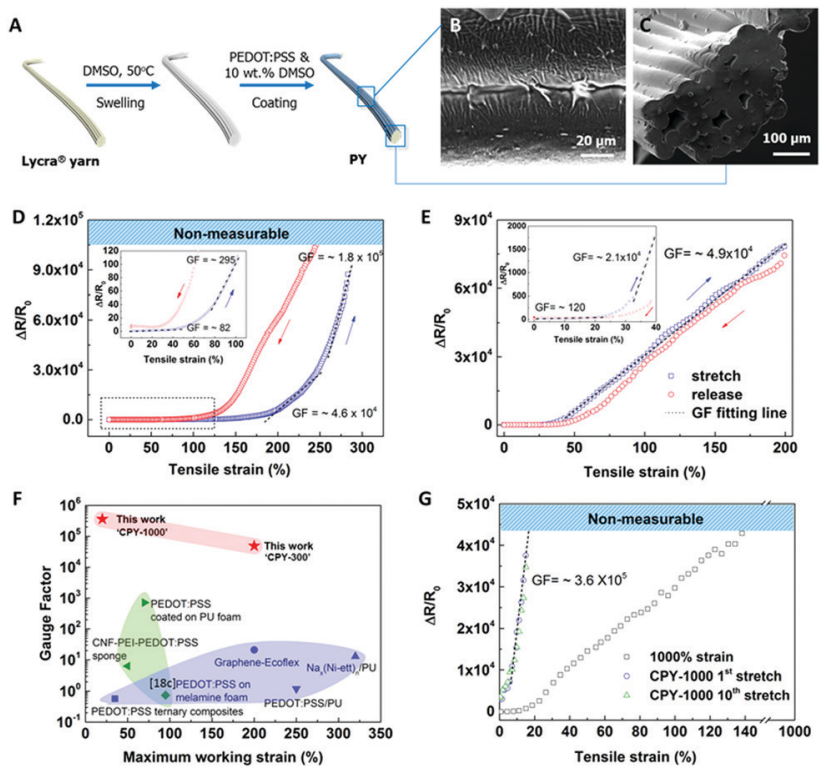

Fig. 1 Fabrication of PEDOT:PSS coated Lycra ${ }^{\circledR}$ yarn, its morphology and sensitivity to strain. (A) Schematic illustration of the 2-step swell-coating procedure for fabricating PEDOT:PSS coated Lycra ${ }^{\mathbb{R}}$ yarn (PY). (B) Surface and $(C)$ cross-sectional SEM images of PY, showing the wrinkled PEDOT coating the yarn as a result of shrinking. Resistance change and the corresponding gauge factor of: (D) the 1st time strain cycle of PY (to $300 \%$ max strain) and (E) the 6th strain cycle of CPY-300 (cracked PY at $300 \%$ pre-strain) up to a $200 \%$ max strain, with low strain values in insets. (F) The summary of the maximum working strain and GF of self-powered strain sensors in literature, $32,34,35,61-64$ compared with this work's results. (G) Resistance changes of $1000 \%$ strain and the presentative 1st and 10th time resistance change with strain of CPY-1000.

Kelly-Tyson and Agrawal-Raj model (detailed in Note S1, $\mathrm{ESI}^{\dagger}{ }^{37-40}$ ). The coating process was optimized, particularly in relation to the electrical properties, by investigating a number of processing parameters ranging from PEDOT:PSS concentration, to coating times and number of coating layers, as described in details in Note S2 (ESI $\dagger$ ). The optimized Lycra ${ }^{\mathbb{R}}$ yarn coated by PEDOT:PSS, is defined as PY and is used as the basis for the following investigations. PY was measured to have an electrical resistivity of $330 \pm 35 \Omega \mathrm{cm}^{-1}$ and $\sim 0.4 \mathrm{wt} \%$ PEDOT:PSS overall content (calculated as in Note S3, $\mathrm{ESI}^{41,42}$ ).

The study of the electrical properties of the coated yarns during deformation needs further attention as it is pivotal for the strain sensing performance. Upon stretching, an exponential increase in the PY electrical resistance is observed (Fig. 1D), with a large variation in the GF values - defined as the slope of the relative change in electrical resistance versus strain curve:

$$
\mathrm{d}\left(\Delta R / R_{0}\right) / \mathrm{d}(\varepsilon),
$$

where the $\Delta R$ is the corresponding resistance variation with strain $(\varepsilon)$, and the initial resistance $\left(R_{0}\right)$ refers to the yarn's resistance at $0 \%$ strain. The GF starts from a value of less than $3.0 \times 10^{2}$, in the strain range of $80-100 \%$, to reach a high value of up to $1.8 \times 10^{5}$ when the strain is between $260 \%$ and $300 \%$ of the same loading cycle. This is due to the fragmentation of the conductive coating layer upon tensile loadings (Fig. S6, ESI†). 
Considering the rigid nature and low strain-to-break of PEDOT:PSS coating, it is expected that this conductive coating layer will start to crack at very low strain (i.e. $<5 \%$ ), as evidenced by the irreversible resistance change upon unloading and by optical microscopy pictures (Fig. S6a, ESI $\dagger$ ).

This PY yarn, after unloading from a $300 \%$ strain, is termed CPY-300 (crack-induced PY subjected to a 300\% strain). After unloading from the first cycle up to $300 \%$ strain, the PEDOT:PSS coating layer was cracked, and the Young's modulus of the yarn reduced to approach the modulus of pure Lycra ${ }^{\circledR}$ yarns (Fig. S4 and Table S1, ESI $\dagger$ ). This indicates that the PEDOT:PSS layer has cracked into patches (Fig. S6, ESI $\dagger$ ) smaller than the critical length for mechanical reinforcement and cannot transfer the tensile stress from the yarn substrate, i.e. the load is mainly carried by the polyurethane. The resistance of CPY-300 increases 10 times after a 10 minute relaxation after pre-strain. It worth to note that the resistance of the CPY-300 at $0 \%$ strain has been taken as a new $R_{0}$ for following measurements.

To better understand the sensitivity of CPY-300, subsequent loading cycles with up to $200 \%$ strain were applied. Interestingly, it is found that the strain sensitivity of CPY-300 is much higher than that of the (non-cracked) PY, even in the low strain range, reaching GF of $2.1 \times 10^{4}$, compared to only 82 for PY, at $30-40 \%$ strain (Fig. 1E). This is due to the specific PEDOT:PSS coating patterned morphology created upon first tensile loading. A cracked, but still interconnected, conductive coating layer induces a higher strain sensitivity as even small macroscopic strain can cause a large localized deformation, with conductive PEDOT:PSS patches separating from each other which, in turn, suddenly increases the electrical resistance. In the strain range of $50 \%$ to $200 \%$, the GF of CPY-300 reaches $4.9 \times 10^{4}, 166$-fold higher than the original PY. It is worth noting that a slightly increasing trend of GF was observed between the second and the fourth tensile loading, attributed to a small refinement of the cracked coating morphology. After the fourth cycle, the morphology becomes invariant and the resistance change with strain becomes stable and reversible (Fig. S5A, ESI $\dagger^{43-60}$ ). The obtained CPY shows an unprecedented combination of properties, including an outstanding sensitivity to deformation together with a very wide strain sensing window. CPY-300 fills the long existing gap in strain sensors possessing high sensitivity and high deformation range, simultaneously (Fig. S5B, ESI $\dagger$ ). Compared to the sensitivity values from literatures, ${ }^{32,34,35,61-64}$ our CPYs show the highest GF values ever reported for self-powered strain sensors (Fig. 1F) together with an excellent maximum working strain.

To study the effect of a different pre-strain, the CPY has been further stretched, up to $1000 \%$ strain (Fig. 1G). This new sample, named CPY-1000, shows a 3000-fold higher strain sensitivity (GF: $\sim 3.6 \times 10^{5}$ ), compared to CPY-300, and good reversibility. Due to the relatively higher electrical resistance, however, the measurable range of CPY-1000 is limited to $20 \%$ strain. The superior sensitivity of CPY-1000 is attributed to a further refinement of the coating morphology, with more loosely connected conductive PEDOT:PSS patches and the presence of secondary cracks (Fig. S6, ESI $\dagger$ ) induced by this large pre-strain. A more detailed description of the mechanism of crack patterns formation and its effect on sensitivity, can be found in Note S4 (ESI $\dagger$ ), where a more comprehensive series of CPY were prepared, with pre-strains ranging from 5\% to $100 \%$, and characterized for their morphological, mechanical, electrical and impedance spectroscopic properties. The impedance spectroscopy of a representative PY and CPY reveal a pure resistive behaviour dominating in the beginning. With strain increasing, cracks partially disconnect the conductive patches from each other, developing capacitor-like behaviour. After prestretched, high strain $(\geq 50 \%)$ will introduce a combined behaviour, i.e., an equivalent circuit of two resistor and capacitor parallel combinations, connected in series. Importantly, we found a reproducible relationship between the pre-strain, the crack morphology and subsequent electrical impedance that enables us to program the electrical properties of the yarn. Clearly, the different pre-strain (e.g. $300 \%$ or $1000 \%$ ) is a simple and efficient parameter to tune both sensitivity and deformation range, which could be programmed in view of a different final application.

Thermoelectricity provides potentially a robust and reliable mechanism to harvest sufficient energy to power our sensing yarns. As expected from the presence of PEDOT:PSS coating, the yarns show a typical p-type thermoelectric behaviour: within the temperature range $210 \mathrm{~K}$ to $360 \mathrm{~K}$ (Fig. 2A), the positive Seebeck coefficient $(\alpha)$ shows a small increase from $11 \mu \mathrm{V} \mathrm{K}^{-1}$ to $15 \mu \mathrm{V} \mathrm{K}^{-1}$ and the resistivity decreases by half, which are in line with properties reported in literature for PEDOT:PSS films treated by DMSO. ${ }^{35}$ The Seebeck coefficient of CPY remains invariant compared to PY, even though the induced cracks in the coating increase the resistivity 2500 -fold after $300 \%$ prestrain.
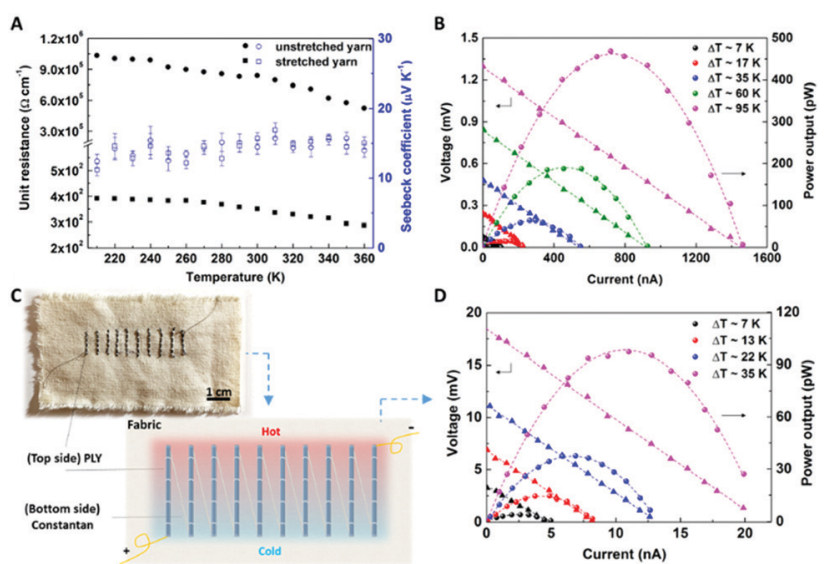

Fig. 2 Thermoelectric properties of the PY. (A) Unit length $(1 \mathrm{~cm})$ resistance and Seebeck coefficient of stretched and unstretched PY, under temperature sweep from $200 \mathrm{~K}$ to $360 \mathrm{~K}$. (B) Power output (circle) and voltage output (triangle) of $2 \mathrm{~cm}$ long PY under different temperature gradients, when various external loads are applied. (C) The photograph and the illustration of the 10 pair legs fabric-based thermoelectric device, and its (D) power output (circle) and voltage output (triangle) under different temperature gradients. 
When the PY is connected to a variable external load resistance, the voltage and power output can be measured under various temperature differences $(\Delta T)$. The open-circuit voltage $U_{\text {open }}\left(U_{\text {open }}=\alpha \Delta T\right)$ is expected to increase linearly with the temperature difference between the ends of the sample (Fig. 2B). The maximum power output $P_{\max }\left(=U^{2} / 2 R=\Delta T^{2} \alpha^{2} / 2 R\right.$, where $R$ is the internal resistance of the yarn) should instead increase with the square of $\Delta T$. It can be observed that a single yarn with a length of $\sim 1.5 \mathrm{~cm}$, under a temperature gradient of $35 \mathrm{~K}$, can generate a power of $60 \mathrm{pW}$. With the temperature difference increasing to $95 \mathrm{~K}, 1.3 \mathrm{mV}$ can be generated, together with a maximum power output of $480 \mathrm{pW}$. The current power output level is promising but only if a multi-'leg' fabric-like device can be produced, with both p- and n-type yarns and low enough internal resistance. A basic device design, shown in Fig. 2C, is manufactured by sewing 10 PYs as p-type legs and constantan wires acting as both n-type 'leg' and electrical connections. PYs and constantan wires were connected in series via knotting the constantan wire on the PY's ends. Such a thermoelectric device, with 10 pairs of 'legs', can generate $18 \mathrm{mV}$ and $105 \mathrm{pW}$ under a temperature gradient of only $35 \mathrm{~K}$ (Fig. 2D).

Our ultra-sensitive CPY smart yarns could then potentially be used in various multi-functional applications such as wireless strain sensors as well as self-powered strain sensors. When connected with external power, the CPY-300 works as a strain sensor with wide sensing range of strain and ultrahigh sensitivity. A clear resistance change can be observed both at large strains, i.e. $200 \%$, tested at $100 \% \mathrm{~min}^{-1}$ strain rate (Fig. 3A-i), and low strain strains, i.e. $1 \%$, tested at $10 \% \mathrm{~min}^{-1}$ strain rate (Fig. 3A-ii). The strain rate has been selected taking in consideration a constant data acquisition rate of 1 point per second. The effect of tensile strain rate (from $5 \% \mathrm{~min}^{-1}$ to $150 \% \mathrm{~min}^{-1}$ ) is to slightly increase the sensitivity from GF 120 to 165 , which is consistent with the viscoelastic response of Lycra ${ }^{\circledR}$ (Fig. S7, ESI $\dagger$ ). The lowest strain, 1\%, gave a 2 -fold resistance change, which corresponds to a GF of 200 as shown in the insert of Fig. 3A-ii. As a $10 \mathrm{~mm}$ yarn has been used for this test, the strain detection limit of CPY-300 is $\ll 0.1 \mathrm{~mm}$. And the response time is in the order of tens of milliseconds, if not

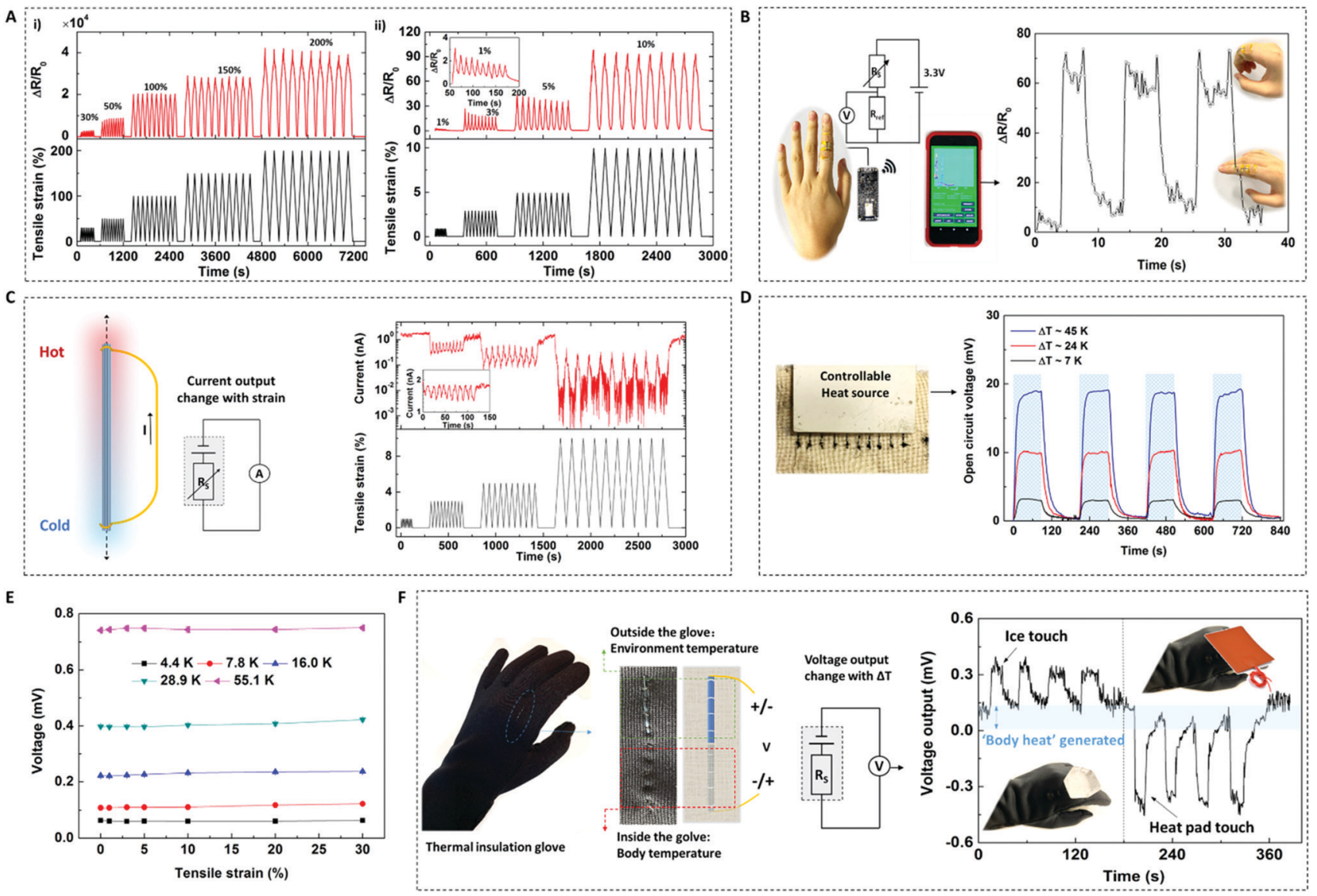

Fig. 3 Demonstration of the CPY applications. An externally powered strain sensor (A) under (i) high and (ii) low strain \& strain rate, showing ultrahigh sensitivity with a wide operation window. Demonstration of CPY working as (B) wireless strain sensor by connecting with Bluetooth ${ }^{\mathbb{R}}$, and (C) selfpowered strain sensor by collecting the short circuit current output under small strains (with $10 \% \mathrm{~mm}^{-1}$ strain rate) and under constant temperature difference $(\Delta T \sim 20 \mathrm{~K})$. (D) Demonstration of CPY working as self-powered temperature (gradient) sensor: the open circuit voltage output at different temperature differences with different strains, and the $(E)$ influence of different strains onto thermal voltage output, measured in open circuit. (F) Demonstration of a smart glove with CPY applied as energy generator by body heat and self-powered temperature sensor, with the temperature of heat pad is $60^{\circ} \mathrm{C}$. 
smaller (Fig. S13A and C, ESI $\dagger$ ). Based on the wide sensing range, this strain sensor could be used in wearable devices for body motion monitoring, as demonstrated for example in Fig. 3B. The sensing signal $\left(\Delta R / R_{0}\right)$, which increases synchronously to the bending of the index finger, can be transmitted wirelessly in real-time to a mobile phone.

In absence of an external power supply, the CPY can also function as a self-powered sensor upon application of an ambient temperature gradient. When the CPY is connected in short circuit (Fig. 3C), the current output will change with strain, as a consequence of the CPY's resistance change. A thermal current is developed in the circuit at a temperature gradient of $20 \mathrm{~K}$. This constant temperature difference along the film generates a thermal voltage $(U)$, which is independent of the strain. Therefore, the current $I(\varepsilon)=U / R(\varepsilon)$ is inversely proportional to its resistance $(R)$. Fig. 3C shows the output current signal change as a function of the imposed strain, demonstrating the ability to sense and generate thermoelectric power concurrently.

Based on its intrinsic thermoelectric properties, the CPY can also be used as a temperature (gradient) sensor. A variable temperature on one end of the CPY can be detected by a change in voltage (Fig. 3D), with a constant reference temperature (e.g. room temperature) at the other end of the yarn, so that CPY could supply different voltages accordingly. The response time for a $7 \mathrm{k}$ gradient is smaller than $23 \mathrm{~s}$ (Fig. S13B, ESI $\dagger$ ). However, for multi-sensor applications, in general, it is important to be able to discriminate the signals induced by different stimuli. In our case, cross-sensitivity can be tackled easily by testing open circuit voltage for temperature signals, and short circuit current for the strain signals, as for the circuit diagrams in Fig. 3C and F. The effect of the temperature gradient variation on the short circuit current $\left(I_{\mathrm{T}}\right)$ could be calculated as $I_{\mathrm{T}}=U / R_{\mathrm{i}}$, where the $R_{\mathrm{i}}$ is the resistance of the yarn. $I_{\mathrm{T}}$ can be easily subtracted from the total current signals $\left(I=I_{\mathrm{T}}+I_{\mathrm{D}}\right)$ to derive the deformation stimuli $\left(I_{\mathrm{D}}\right)$ - hence tackling the cross-sensitivity. Meanwhile, strains have no influence to the voltage output, which is also an essential requirement for multi-functional and self-powered strain sensors. The voltage output $(U)$, i.e. open circuit voltage, has been measured under different strains (Fig. 3E). This voltage is constant, at the same temperature difference, for any strain between $0 \%$ and $30 \%$. Here it is worth to be noted, due to the ultrahigh resistance sensitive to the strain, the open circuit voltage can only be measured accurately below 30\% strains when the resistance of the voltage meter cannot be seen as 'open' compared to resistance of the yarn. This demonstrates that, within this strain range, the CPY thermoelectric voltage output signal only depends on the temperature difference. This is because the voltage output is only relying on the temperature difference $(\Delta T)$ between the two ends of the composite yarn as determined by equation $U=S \Delta T$, where the Seebeck coefficient $(S)$ of the coated yarn is kept at $\sim 15 \mu \mathrm{V} \mathrm{K}^{-1}$ (typical value of the DMSO doped PEDOT:PSS films ${ }^{35}$ ). The Seebeck coefficient is not affected by the cracks (Fig. 2A). Upon stretching, the overall temperature difference $\left(\Delta T=T_{\mathrm{h}}-T_{\mathrm{c}}\right)$ is constant and defined by the hot end $\left(T_{\mathrm{h}}\right)$ and room temperature $\left(T_{\mathrm{c}}\right)$. Hence, CPY can be considered as a self-powered temperature (gradient) sensor, with the voltage output as the data acquired. The relationship between open circuit voltage and temperature difference is linear $(U=0.42 \times \Delta T)$, with an overall Seebeck coefficient for the 10 couples defined by the slope of the curve (Fig. S14, ESI $\dagger$ ). Besides, the sensing performance of the sensor is stable under different ambient temperatures (at least between $210 \mathrm{~K}$ and 360 K, Fig. 2A), and humidity (relative humidity between $20 \%$ and $80 \%$, Fig. S15, ESI $\dagger$ ).

As a proof of concept, a 'smart' glove has been fabricated by sewing CPY through the fabric of a thermally insulated glove and fixing both sides (Fig. 3F). Electrodes were embedded by knotting thin copper wire at the ends. The connection between the yarn and electrodes was enhanced by carbon grease and ethylcyanoacrylate (Loctite ${ }^{\circledR}$, Henkel). In such configuration, the hand temperature is kept constant by the glove, and can then be considered as the reference temperature of the CPY sensor. Therefore, the ambient temperature or the temperature of an object in contact with the glove can be sensed. In a different embodiment, a CPY can harvest body heat and power a second CPY strain sensor. Considering the small temperature difference $\left(\Delta T \sim 10{ }^{\circ} \mathrm{C}\right)$ normally existing between human body and the environment, it can be estimated that $1.8 \mathrm{k}$ couples of PYs and constantan wires with $5 \mathrm{~mm}$ length are required to reach a power output of $10 \mu \mathrm{W}$, which is normally the requirements for operating wearable electronics (Note S5, ESI $\dagger$ ).

These results demonstrate that CPY could be used as multifunctional self-powered sensors, with both strain and temperature detection, energy harvesting, and physical properties such as high deformability and water resistance suitable for a wide range of applications.

\section{Conclusions}

In conclusion, we report a self-powered, ultra-sensitive strain and temperature sensor based on PEDOT:PSS coated Lycra ${ }^{\circledR}$ yarn, prepared by a simple swell-coating method. The ultrahigh sensitivity to strain (GF $\sim 49000$ at $50 \%$ to $200 \%$ ) and large stretchability (up to $1000 \%$ ) provide a unique combination of properties addressing the long existing challenge in strain sensors, with the highest value achieved in both sensitivity and stretchability simultaneously. By controlling the pre-strain value to induce crack patterns of the conductive PEDOT:PSS layer on Lycra ${ }^{\circledR}$, the sensitivity can be adjusted for different target values. The thermoelectric properties of the PEDOT:PSS has been preserved, with a Seebeck coefficient of $15 \mu \mathrm{V} \mathrm{K}$ after stretching, providing temperature sensing ability (regardless of deformation) and/or energy harvesting for self-powered sensing. The open circuit voltage signal can be used to detect temperature variations, while the short circuit current can be used to sense mechanical deformations.

Our sensing yarns have great potential for various applications, especially wearable electronics. A wireless movements' detector and a proof-of-concept glove, which can harvest body energy and sense the environment and object temperature 
in a self-powered mode, have been developed as simple demonstrators.

Looking into further developments, one challenge to further broaden the practical applicability will be to increase the power output. The power harvested by thermoelectricity ( $\sim 60 \mathrm{pW}$ under $\Delta T \sim 35 \mathrm{~K}$ for 1 yarn) is, for instance, still insufficient to operate commercial data transfer systems ( $\sim 10 \mu \mathrm{W}$ typically required). The development of more efficient organic thermoelectric materials, particularly n-type, could be a potential strategy for reaching high TE powers. Alternative energy harvesting mechanisms, like piezoelectricity and triboelectricity, perhaps in combination with flexible energy storage solutions, could also yield promising results.

\section{Author contributions}

K. W. performed all the experimental works on yarn fabrication, devices fabrication, characterizations, and data analysis. K. W. and Y. L. performed the smart glove sensing measurements with help from G. S. K. W. and G. B. performed the wireless sensing fabrication and measurements after discussion with F. G. and E. B. K. W. and P. T. G. performed the water stabilization measurements. Yarns fabrication was discussed with H. Z., interfacial shear strength and thickness calculation was discussed with D. G. P. and E. B. Impedance analysis and equivalent circuit fitting were discussed with S. K. and E. B. and suggested by M. B. This work was ideated and supervised by E. B. The paper was written with contributions from all authors with suggestions by C. W. M. B. and O. F.

\section{Conflicts of interest}

There are no conflicts to declare.

\section{Acknowledgements}

K. W. thanks Chinese Scholarship Council (CSC) for financial support. O. F. is supported by a Royal Society University Research Fellowship (UF140372 \& UF URF/R/201013).

\section{Notes and references}

1 M. Baumgartner, F. Hartmann, M. Drack, D. Preninger, D. Wirthl, R. Gerstmayr, L. Lehner, G. Mao, R. Pruckner, S. Demchyshyn, L. Reiter, M. Strobel, T. Stockinger, D. Schiller, S. Kimeswenger, F. Greibich, G. Buchberger, E. Bradt, S. Hild, S. Bauer and M. Kaltenbrunner, Nat. Mater., 2020, 19, 1102-1109.

2 D. Zhang, S. Xu, X. Zhao, W. Qian, C. R. Bowen and Y. Yang, Adv. Funct. Mater., 2020, 30, 1910809.

3 E. Torres Alonso, D. P. Rodrigues, M. Khetani, D.-W. Shin, A. De Sanctis, H. Joulie, I. de Schrijver, A. Baldycheva, H. Alves, A. I. S. Neves, S. Russo and M. F. Craciun, $n p j$ Flexible Electron., 2018, 2, 25.

4 S. S. Kwak, H.-J. Yoon and S.-W. Kim, Adv. Funct. Mater., 2019, 29, 1804533.
5 A. Chortos, J. Liu and Z. Bao, Nat. Mater., 2016, 15, 937-950.

6 C. M. Boutry, M. Negre, M. Jorda, O. Vardoulis, A. Chortos, O. Khatib and Z. Bao, Sci. Robot., 2018, 3, eaau6914.

7 W. Gao, S. Emaminejad, H. Y. Y. Nyein, S. Challa, K. Chen, A. Peck, H. M. Fahad, H. Ota, H. Shiraki, D. Kiriya, D.-H. Lien, G. A. Brooks, R. W. Davis and A. Javey, Nature, 2016, 529, 509-514.

8 C. Tan, Z. Dong, Y. Li, H. Zhao, X. Huang, Z. Zhou, J.-W. Jiang, Y.-Z. Long, P. Jiang, T.-Y. Zhang and B. Sun, Nat. Commun., 2020, 11, 3530.

9 Y. Yamamoto, S. Harada, D. Yamamoto, W. Honda, T. Arie, S. Akita and K. Takei, Sci. Adv., 2016, 2, e1601473.

10 E. O. Polat, G. Mercier, I. Nikitskiy, E. Puma, T. Galan, S. Gupta, M. Montagut, J. J. Piqueras, M. Bouwens, T. Durduran, G. Konstantatos, S. Goossens and F. Koppens, Sci. Adv., 2019, 5, eaaw7846.

11 C. Wang, K. Xia, H. Wang, X. Liang, Z. Yin and Y. Zhang, Adv. Mater., 2019, 31, 1801072.

12 J. A. Lee, A. E. Aliev, J. S. Bykova, M. J. de Andrade, D. Kim, H. J. Sim, X. Lepró, A. A. Zakhidov, J. B. Lee and G. M. Spinks, Adv. Mater., 2016, 28, 5038-5044.

13 S.-J. Park, J. Kim, M. Chu and M. Khine, Adv. Mater. Technol., 2016, 1, 1600053.

14 Y. Khan, A. E. Ostfeld, C. M. Lochner, A. Pierre and A. C. Arias, Adv. Mater., 2016, 28, 4373-4395.

15 L. K. Randeniya, P. J. Martin, A. Bendavid and J. McDonnell, Carbon, 2011, 49, 5265-5270.

16 T. J. Jia, Y. Wang, Y. Y. Dou, Y. W. Li, M. J. de Andrade, R. Wang, S. L. Fang, J. J. Li, Z. Yu, R. Qiao, Z. J. Liu, Y. Cheng, Y. W. Su, M. Minary-Jolandan, R. H. Baughman, D. Qian and Z. F. Liu, Adv. Funct. Mater., 2019, 29, 1808241.

17 W. He, C. Wang, H. Wang, M. Jian, W. Lu, X. Liang, X. Zhang, F. Yang and Y. Zhang, Sci. Adv., 2019, 5, eaax0649.

18 W. Li, F. J. Xu, L. J. Sun, W. Liu and Y. P. Qiu, Sens. Actuators, $B, 2016,230,528-535$.

19 Y. Wang, J. Hao, Z. Huang, G. Zheng, K. Dai, C. Liu and C. Shen, Carbon, 2018, 126, 360-371.

20 B. Park, J. Kim, D. Kang, C. Jeong, K. S. Kim, J. U. Kim, P. J. Yoo and T.-i. Kim, Adv. Mater., 2016, 28, 8130-8137.

21 T. Yamada, Y. Hayamizu, Y. Yamamoto, Y. Yomogida, A. Izadi-Najafabadi, D. N. Futaba and K. Hata, Nat. Nanotechnol., 2011, 6, 296-301.

22 Y. Zheng, Y. Li, K. Dai, Y. Wang, G. Zheng, C. Liu and C. Shen, Compos. Sci. Technol., 2018, 156, 276-286.

23 M. Kasimatis, E. Nunez-Bajo, M. Grell, Y. Cotur, G. Barandun, J.-S. Kim and F. Güder, ACS Appl. Mater. Interfaces, 2019, 11, 47577-47586.

24 Y. Jiang, Z. Liu, N. Matsuhisa, D. Qi, W. R. Leow, H. Yang, J. Yu, G. Chen, Y. Liu, C. Wan, Z. Liu and X. Chen, Adv. Mater., 2018, 30, 1706589.

25 X. Liao, Z. Zhang, Z. Kang, F. Gao, Q. Liao and Y. Zhang, Mater. Horiz., 2017, 4, 502-510.

26 W. Weng, P. Chen, S. He, X. Sun and H. Peng, Angew. Chem., Int. Ed., 2016, 55, 6140-6169.

27 J. Chen, Y. Huang, N. Zhang, H. Zou, R. Liu, C. Tao, X. Fan and Z. L. Wang, Nat. Energy, 2016, 1, 16138. 
28 N. Zhang, J. Chen, Y. Huang, W. Guo, J. Yang, J. Du, X. Fan and C. Tao, Adv. Mater., 2016, 28, 263-269.

29 N. Soin, T. H. Shah, S. C. Anand, J. Geng, W. Pornwannachai, P. Mandal, D. Reid, S. Sharma, R. L. Hadimani and D. V. Bayramol, Energy Environ. Sci., 2014, 7, 1670-1679.

30 H. Guo, X. Pu, J. Chen, Y. Meng, M.-H. Yeh, G. Liu, Q. Tang, B. Chen, D. Liu, S. Qi, C. Wu, C. Hu, J. Wang and Z. L. Wang, Sci. Robot., 2018, 3, eaat2516.

31 X. Pu, H. Guo, J. Chen, X. Wang, Y. Xi, C. Hu and Z. L. Wang, Sci. Adv., 2017, 3, e1700694.

32 D. Zhang, K. Zhang, Y. Wang, Y. Wang and Y. Yang, Nano Energy, 2019, 56, 25-32.

33 S. J. Kim, J. H. We and B. J. Cho, Energy Environ. Sci., 2014, 7, 1959-1965.

34 K. Wan, P. J. Taroni, Z. Liu, Y. Liu, Y. Tu, G. Santagiuliana, I.-C. Hsia, H. Zhang, O. Fenwick, S. Krause, M. Baxendale, B. C. Schroeder and E. Bilotti, Adv. Electron. Mater., 2019, 5, 1900582.

35 P. J. Taroni, G. Santagiuliana, K. Wan, P. Calado, M. Qiu, H. Zhang, N. M. Pugno, M. Palma, N. Stingelin-Stutzman, M. Heeney, O. Fenwick, M. Baxendale and E. Bilotti, Adv. Funct. Mater., 2018, 28, 1704285.

36 Y. Tao, Y. Liu, H. Zhang, C. A. Stevens, E. Bilotti, T. Peijs and J. J. C. Busfield, Compos. Sci. Technol., 2018, 167, 24-31.

37 T. Ohsawa, A. Nakayama, M. Miwa and A. Hasegawa, J. Appl. Polym. Sci., 1978, 22, 3203-3212.

38 S. F. Zhandarov and E. V. Pisanova, Mech. Compos. Mater., 1996, 31, 325-336.

39 U. Lang, N. Naujoks and J. Dual, Synth. Met., 2009, 159, 473-479.

40 S. Zhang, Biological and Biomedical Coatings Handbook: Applications, CRC Press, 2016.

41 U. Lang, N. Naujoks and J. Dual, Synth. Met., 2009, 159, 473-479.

42 Z.-H. Ge, Y. Chang, F. Li, J. Luo and P. Fan, Chem. Commun., 2018, 54, 2429-2431.

43 C. S. Boland, U. Khan, C. Backes, A. O'Neill, J. McCauley, S. Duane, R. Shanker, Y. Liu, I. Jurewicz, A. B. Dalton and J. N. Coleman, ACS Nano, 2014, 8, 8819-8830.

44 S. Gong, D. T. H. Lai, Y. Wang, L. W. Yap, K. J. Si, Q. Shi, N. N. Jason, T. Sridhar, H. Uddin and W. Cheng, ACS Appl. Mater. Interfaces, 2015, 7, 19700-19708.

45 M. Amjadi, Y. J. Yoon and I. Park, Nanotechnology, 2015, 26, 375501 .
46 X. Xiao, L. Yuan, J. Zhong, T. Ding, Y. Liu, Z. Cai, Y. Rong, H. Han, J. Zhou and Z. L. Wang, Adv. Mater., 2011, 23, 5440-5444.

47 D. Kang, P. V. Pikhitsa, Y. W. Choi, C. Lee, S. S. Shin, L. Piao, B. Park, K.-Y. Suh, T.-i. Kim and M. Choi, Nature, 2014, 516, 222-226.

48 N. Lu, C. Lu, S. Yang and J. Rogers, Adv. Funct. Mater., 2012, 22, 4044-4050.

49 E. Roh, B.-U. Hwang, D. Kim, B.-Y. Kim and N.-E. Lee, ACS Nano, 2015, 9, 6252-6261.

50 Y. R. Jeong, H. Park, S. W. Jin, S. Y. Hong, S.-S. Lee and J. S. Ha, Adv. Funct. Mater., 2015, 25, 4228-4236.

51 C. Mattmann, F. Clemens and G. Tröster, Sensors, 2008, 8, 3719-3732.

52 S. Gong, D. T. H. Lai, B. Su, K. J. Si, Z. Ma, L. W. Yap, P. Guo and W. Cheng, Adv. Electron. Mater., 2015, 1, 1400063.

53 J. T. Muth, D. M. Vogt, R. L. Truby, Y. Mengüç, D. B. Kolesky, R. J. Wood and J. A. Lewis, Adv. Mater., 2014, 26, 6307-6312.

54 J.-H. Kong, N.-S. Jang, S.-H. Kim and J.-M. Kim, Carbon, 2014, 77, 199-207.

55 B.-U. Hwang, J.-H. Lee, T. Q. Trung, E. Roh, D.-I. Kim, S.W. Kim and N.-E. Lee, ACS Nano, 2015, 9, 8801-8810.

56 M. Amjadi, A. Pichitpajongkit, S. Lee, S. Ryu and I. Park, ACS Nano, 2014, 8, 5154-5163.

57 M. Amjadi, K.-U. Kyung, I. Park and M. Sitti, Adv. Funct. Mater., 2016, 26, 1678-1698.

58 S. Han, C. Liu, X. Lin, J. Zheng, J. Wu and C. Liu, ACS Appl. Polym. Mater., 2020, 2, 996-1005.

59 J. Wu, Z. Wu, X. Lu, S. Han, B.-R. Yang, X. Gui, K. Tao, J. Miao and C. Liu, ACS Appl. Mater. Interfaces, 2019, 11, 9405-9414.

60 Z. Duan, Y. Jiang, S. Wang, Z. Yuan, Q. Zhao, G. Xie, X. Du and H. Tai, ACS Sustainable Chem. Eng., 2019, 7, 17474-17481.

61 F. Zhang, Y. Zang, D. Huang, C.-a. Di and D. Zhu, Nat. Commun., 2015, 6, 8356.

62 H. Cheng, Y. Du, B. Wang, Z. Mao, H. Xu, L. Zhang, Y. Zhong, W. Jiang, L. Wang and X. Sui, Chem. Eng. J., 2018, 338, 1-7.

63 J. Oh, J.-H. Kim, K. T. Park, K. Jo, J.-C. Lee, H. Kim and J. G. Son, Nanoscale, 2018, 10, 18370-18377.

64 S. Kee, M. A. Haque, D. Corzo, H. N. Alshareef and D. Baran, Adv. Funct. Mater., 2019, 29, 1905426. 